\title{
PROBABILITY IN QUANTUM MECHANICS
}

\author{
J.G. GILSON \\ Department of Applied Mathematics \\ Queen Mary College \\ London, E1 4NS England \\ (Received September 12, 1980 and in revised form February 17, 1981)
}

ABSTRACT. By using a fluid theory which is an alternative to quantum theory but from which the latter can be deduced exactly, the long-standing problem of how quantum mechanics is related to stochastic processes is studied. It can be seen how the Schröinger probability density has a relationship to time spent on small sections of an orbit, just as the probability density has in some classical contexts. KEY WORDS. Quantum theory, Schrödinger equation, Probability density. Fluid theary, Stochastic process.

1980 MATHEMATICS SUBJECT CLASSIFICATION CODES. $76405,80 A 15,81 A 12$.

1. INTRODUCTION.

The long-standing question of whether quantum mechanics is a stochastic process [1] has been studied by many researchers $[2,3,4,5,6] ;[3,4,5,6]$ constitute just a few of the many attempts to show that quantum mechanics has a stochastic basis. Of course a probability density, $\rho(x, t)$, varying with position $x$ and possibly with time $t$, occurs in quantum theory. If stochastic process is defined broadly as "a process describable by probabilities, possibly time dependent", then quantum mechanics is a stochastic process. However, probability in Schrodinger quantum theory seems at first ad hoc:

$$
\rho=\psi^{*}(x, t) \psi(x, t),
$$

where $\psi$ is the 'wave function'. This connection between $\rho$ and $\psi$, even after considerable acquaintance, lacks content; for one thing, $\psi$ itself lacks physical 
interpretation. $\psi$ is essentially a mathematical auxiliary, though of great computational value. Thus even with the broad definition of stochastic process, doubts about the probabilistic status of $\rho$ fuzz the issue. The favourite stochastic process thought possibly to underlie quantum mechanics is Brownian motion [4] or some variant of Brownian motion [5]. The reason for this direction of the stochastic search is the analogy between the shape of the simple Schrodinger equation,

$$
-i \hbar \frac{\partial \psi}{\partial t}=\frac{h^{2}}{2 m} \frac{\partial^{2} \psi}{\partial x^{2}},
$$

and the equation for Brownian motion,

$$
\frac{\partial \rho}{\partial t}=D \frac{\partial^{2} \rho}{\partial x^{2}}
$$

However, the $i$ in (1.2) much weakens this 'analogy'. As things stand now, Brownian motion might conceivably be made into a basis for quantum mechanics; still, this seems unlikely despite some substantial theoretical elaboration [3]. However, starting from an alternative fluid version for the one dimensional Schrodinger equation, a connection with the stochastic point of view will be shown in this paper. But the process involved is not Brownian motion.

Now, fluid equations are deterministic. But fluids can be considered to be composed of particles, part of whose motion is known only probabilistically. When averages are taken over this stochastic or probabilistic part of the 'particle' motion, the exact deterministic 'fluid' motion or change of state becomes known; the particle motion need not be steady. Suppose a position $(x, y)$ is given in a two dimensional space. It will be assumed that particles can be found rotating around points distant from $(x, y)$ but with a range of different possible angular velocities about these distant points which may themselves have local translational velocities. The basic stochastic feature is taken to be that the probability for finding a particle with any specific value of angular velocity $\omega$ about a distant point is given by a distribution function $f(\omega)$. However, in full, there is a distribution function for $w$ at each position $(\mathrm{x}, \mathrm{y})$ which can also change with time, $f(\omega)=f(\omega \mid x, y, t)$. Fluid equatiuns are usually regarded as being deterministic even when they involve the thermodynamical properties associated with heat and compressibility. 
Such fluid equations to be used here, refer to quantities that are averages. The stochastic process is a substructure upon which deterministic averages are constructed just as is done for example in the kinetic theory of gases when probabilistic ideas about particles and their states of motion are used to deduce the deterministic fluid or thermodynamical equations. After taking averages, uncertainty becomes replaced by determinism, not necessarily for the individual particles but for the fluid viewed as a composite structure or as a macroscopic entity. However, the stochastic character of the processes underlying thermodynamics and related areas is still considered to be an operative explanation of the form and the properties of the macroscopic quantities and equations involved and it is in this sense that stochastic ideas are being used in this paper. Even orthodox Schrodinger quantum mechanics is deterministic with respect to the time evolution of the wave function. Determinism becomes fogged in quantum mechanics when one attempts to track or con-sider the detailed movements of individual particles along specific paths and indeed this is also the case with classical thermodynamics and to a lesser extent with classical hydrodynamics.

In this paper, we shall also make use of the auxiliary idea that very close to the centre of a mobile point vortex 'particles' move in circular orbits which have a rather simple character, somewhat like the deterministic orbits of classical point particles in motion round a force centre. We shall not need to know the position of a particle on such an orbit at each instant of time but rather we only need work with the probability for finding a particle in small section ds of the trajectory.

The relation between the one dimensional schrodinger equation and a two dimensional fluid has been given in some detail elsewhere [7]. Here we are more concerned with the generation of the fluid structure from a stochastic basis and this will now be described.

\section{FLUID AND PROBABILITY}

The present author's [7,8] fluid alternative for the one dimensional Schrödinger equation is a flow process taking palce on a two dimensional surface. The two dimensional surface is a real plane having two real orthogonal directions denoted by unit vectors $\underline{i}$ and $\dot{j}$. However, the real surface, when its description is transformed 
into the language of complex functions, becomes a basis for Schrodinger theory with the $\underline{i}, j$ plane corresponding to the analytically continued $x$, coordinate space, of the Schrodinger equation. In place of the Schrodinger probability density $\rho(x, t)$, we have $\rho(x, y, t)$ giving either a mass density distributed over the $\underline{i}, \underline{j}$ plane or a probability density according to the dimensioned constants that occur multiplied into $\rho$.

In classical physics when a single particle moves deterministically on an orbit with a given speed $v(x, y)$, say, for each position $(x, y)$ it occupies in the course of its journey, the time it spends in a small vector region (dx, dy) or small scalar section ds of its trajectory is given by,

$$
\mathrm{dt}=\frac{\mathrm{ds}}{\mathrm{v}(\mathrm{x}, \mathrm{y})},
$$

where $d s^{2}=(d x)^{2}+(d y)^{2}$ in the case of planar motion. Thus if we were in the position of not knowing the initial conditions of the motion for the single particle but in fact only knew its constant value of energy, $E=\frac{1}{2} \mathrm{mv}^{2}+v(x, y)$, say, then, although we would have the speed at which it traverses each orbital position, we would not know the time of passing such a position. Under these conditions it is natural to define a probability for finding the particle between points $s_{1}$ and $s_{2}$ on the orbit by,

$$
\mathrm{P}\left(\mathrm{s}_{1}<\mathrm{s}_{2}<\mathrm{s}_{2}\right)=\frac{1}{\tau} \int_{\mathrm{s}_{1}}^{\mathrm{s}_{2}} \frac{\mathrm{ds}}{\mathrm{v}(\mathrm{x}, \mathrm{y})},
$$

where,

$$
\tau=\oint \frac{\mathrm{ds}}{\mathrm{v}(\mathrm{x}, \mathrm{y})},
$$

is the total time spent traversing the orbit once.

We can use (2.2) and (2.3) to define a normalized probability density $\rho(x, y)$ for the orbit by,

$$
\rho(\mathrm{x}, \mathrm{y})=\frac{1}{\tau \mathrm{v}(\mathrm{x}, \mathrm{y})},
$$

where

$$
\oint \rho(x, y) d s=1,
$$

by (2.3). The thinking behind the introduction of a probability density of the form (2.2) is entirely classical, intuitively satisfying, and is the sort of con- 
nection between kinematics and statistics which makes for a plausible introduction of probability into a dynamical situation. It is not thought that a relation such as (2.4) has any general validity in quantum mechanics as far as connections between the quantum probability density $\rho(x, t)$ and local velocities are concerned. It will now be shown that a relation similar to (2.4) holds rigorously for the equivalent fluid theory form $\rho(x, y, t)$. Let us consider the type of fluid motion taking place on the $\underline{i}, \underline{j}$ surface corresponding to the complex plane of Schrodinger quantum mechanics. Certainly, in general, vorticity is involved [7]. We can form a classical image of the fluid motion as follows. A single point vortex, if it occurs, will move with the average motion of the momentum fluid. We make a distinction between momentum fluid velocity field denoted by $\bar{v}=v_{1} \underline{i}-v_{2} \underline{j}$ and mass fluid velocity field denoted by $\underline{v}=v_{1} \underline{i}+v_{2} \underline{j}$. The reason for the identification of $\overline{\mathrm{v}}=\mathrm{v}_{1} \underline{\mathrm{i}}-\mathrm{v}_{2} \underline{j}$ with the momentum velocity field vector is that one dimensional Schröinger quantum mechanics can be alternatively expressed by a Newtonian equation for motion on a two dimensional surface of the form,

$$
\left.\mathrm{m} \frac{\mathrm{d} \overline{\mathrm{v}}}{\mathrm{d}}\right|_{\underline{\mathrm{v}}}=-\nabla(\mathrm{kT})-\nabla \mathrm{V}_{1}
$$

where,

$$
\left.\frac{\mathrm{d} \overline{\mathbf{v}}}{\mathrm{dt}}\right|_{\underline{\mathbf{v}}}=\frac{\partial \overline{\bar{v}}}{\partial \mathrm{t}}+\underline{\mathbf{v}} \cdot \nabla \underline{\bar{v}}
$$

and $\left.\frac{\mathrm{d} \overline{\mathrm{v}}}{\mathrm{dt}}\right|_{\underline{v}}$ is differentiation of $\underline{\vec{v}}$ following the mass velocity flow given by $\underline{v} \cdot$ $\mathrm{T}$ is a local temperature for the fluid and $\mathrm{V}_{1}$ is the usual external potential function. Clearly $\underline{\bar{v}}$ corresponds to the classical momentum vector. However, we shall not use these equations in this work but instead rather work from first principles. In fact, the Newtonian equation above can easily be derived from the results obtained in this paper.

The momentum flow is purely lamellar flow. Thus given the existence of a point vortex, it will induce, relative to its own frame, the usual external velocity field [9], $v=v / r$, where $\nu$ is its strength. Alternatively, such a point vortex at $(x, y)$, say, induces an angular motion at $\left(x^{\prime}, y^{\prime}\right)$ of magnitude $\lambda=\omega=\nu / r^{2}$ about $(x, y)$ where $r=\left\{\left(x-x^{\prime}\right)^{2}+\left(y-y^{\prime}\right)^{2}\right\}^{1 / 2}$. Thus we can analyse the particle 
motion taking place at $\left(x^{\prime}, y^{\prime}\right)$ in terms of motions of nearby vortices and the angular velocities they induce at $\left(x^{\prime}, y^{\prime}\right)$. We shall now 1 ist the characteristics of the fluid process which will be used in the following pages and which are suggested by the previous discussion.

A. Let $\Gamma$ denote an assembly of point vortices with each member having the same strength $\nu$. We shall assume that the actual vortex fields that occur on the $\underline{i}, \underline{j}$ plane can be formed by some spatial distribution of these vortices.

B. The vortices $\Gamma$ move with the average motion of the momentum fluid and distribute themselves with, in general, a variable density conforming to the dynamical influences at work within the fluid.

C. Each vortex induces velocities and angular velocities in the fluid at places distant from its position.

D. Circular orbits having centres moving with the average momentum velocity field can be found limitingly close in towards a vortex centre.

E. The actual vorticity at a point is twice the average angular velocity induced at that point by the field of mobile vortices $\Gamma$. In other words, the spatialdistribution of vortices $\Gamma$ determine the actual vorticity $\underline{\zeta}=\nabla \wedge \underline{v}$, where $\underline{v}$ is the average mass velocity.

F. On suitable orbits the probability for finding a fluid particle in a small region is inversely proportional to the speed assumed by the particles in that region as in formula (2.4) and, in particular, on the orbits referred to in D.

The six statements above constitute a straight-forward classical and visualisable image of the influences at work throughout the fluid. We need ultimately either to know or to be able to find the function $f(\omega)$ which tells us the distribution (or probability density) for finding an angular velocity $\omega$ at any given value of $\omega$. We can, however, proceed some considerable way without knowing $f(\omega)$ explicitly. It is convenient, in the first instance, to express $f(\omega)$ as a density in $\omega$ space and then in accordance with our assumptions, particularly $\mathrm{C}$ and also if $\omega$ is related to $r$ by $\omega=\nu / r^{2}$ as is normally the case, we can find the probability distribution 
for angular velocity contributions in configuration space. We shall work with the magnitude of angular velocity. Thus we put,

$$
\lambda=|\omega| \geq 0
$$

Hence if $f(\lambda)$ is the probability density for funding an angular velocity with magnitude $\lambda$ in $\lambda$ space, then,

$$
\begin{aligned}
& \int_{0}^{\infty} f(\lambda) d \lambda=1 \\
& \int_{0}^{\infty} \lambda f(\lambda) d \lambda=\bar{\lambda}
\end{aligned}
$$

where $\bar{\lambda}$ is the mean angular velocity. The dependence of $f(\lambda)$ on $x, y$ and $t$ is not at the moment being indicated explicitly. However, in full, $f(\lambda)=f(\lambda \mid x, y, t)$ and consequent ly $\bar{\lambda}=\bar{\lambda}(x, y, t)$. As we are assuming that the local angular velocities are being induced by vortices, a local angular velocity of magnitude $\lambda$ will be induced by a vortex at a distance $r$, where

$$
\lambda r^{2}=\nu \text { and } d \lambda=-\frac{2 \nu}{r^{3}} d r
$$

and $\nu$ is the strength of each individual point vortex in the collection $\Gamma$. Thus the configuration space density, $P(r)$, for the distribution of the vortices in the set $\Gamma$ can be obtained by transforming (2.7) and (2.8) using (2.9). We obtain,

$$
\begin{aligned}
& \int_{0}^{\infty} f\left(\frac{v}{r^{2}}\right) \frac{2 \nu}{r^{3}} d r=1 \\
& \int_{0}^{\infty} \frac{v}{r^{2}} f\left(\frac{\nu}{r^{2}}\right) \frac{2 \nu}{r^{3}} d r=\bar{\lambda}
\end{aligned}
$$

Thus

$$
P(r)=f\left(\frac{\nu}{r^{2}}\right) \frac{2 \nu}{r^{3}}=-f(\lambda) \frac{d \lambda}{d r}
$$

is the probability for finding a vortex of strength $v$ at a distance $r$ from the point of interest. We shall now employ $D$ from the list of characteristics. Let us consider the motion of an individual fluid particle. Such a particle will be moving with the average fluid motion but in addition to that it will have whatever angular velocity is induced at its position by the local vortices which are themselves moving with the average motion of fluid. Let us first consider $\mathrm{x}$ direction 
motion only. According to our principles, we can write down the two values for the total $x$ direction velocity $u_{1}$ for two positions separated by a distance $2 \mathrm{r}$ in the $\mathrm{y}$ direction as follows,

$$
\begin{aligned}
& u_{1}(x, y+r)=v_{1}(x, y)+\lambda r . \\
& u_{1}(x, y-r)=v_{1}(x, y)-\lambda r .
\end{aligned}
$$

(2.13) and (2.14) give the total velocities at the points $(x, y+r)$ and $(x, y-r)$ for particles on an orbit centre $(x, y)$ and radius $r$ for an angular velocity of magnitude $\lambda$. Here we are assuming clockwise rotation with $\mathrm{v}_{1}(\mathrm{x}, \mathrm{y})$ being the average fluid velocity for the $x$ direction at $(x, y)$. From (2.9) and (2.14), we see that

$$
\mathrm{u}_{1}(\mathrm{x}, \mathrm{y}-\mathrm{r})=\mathrm{v}_{1}(\mathrm{x}, \mathrm{y})-\frac{\mathrm{v}}{\mathrm{r}}
$$

and so, for sufficiently small positive values of $r, u_{1}(x, y-r)$ will become negative. Likewise, $u_{1}(x, y+r)$ will be positive for sufficiently small $r$. We can now use the probability time-in-orbit principle (2.4) (or F). We can express this as 'the ratio of the probabilities for finding a particle at two distint points on a circular orbit equals the reciprocal of the ratio ot the corresponding speeds at these points. Thus using the points $(x, y,+r)$ and $(x, y-r)$, we have,

$$
\frac{\rho(x, y+r)}{\rho(x, y-r)}=\frac{\left|u_{1}(x, y-r)\right|}{\left|u_{1}(x, y+r)\right|}
$$

However, from the previous discussion concerning the signs of $u_{1}(x, y \pm r)$, we have,

$$
\left|u_{1}(x, y+r)\right|=+\left(v_{1}(x, y)+\lambda r\right)
$$

and

$$
\left|u_{1}(x, y-r)\right|=-\left(v_{1}(x, y)-\lambda r\right)
$$

for sufficiently small $\mathrm{r}$. Thus from $(2.17)$ and $(2.18)$ with a little rearrangement, we get,

$$
v_{1}=-v \frac{(\rho}{(\rho} \frac{(x, y+r)-\rho(x, y-r)) / 2 r}{(x, y+r)+\rho(x, y-r)) / 2}
$$

where (2.9) has been used to eliminate $\lambda$. If we now go to the limit $r \rightarrow 0$ in accordance with the assumed characteristic $D$ in order to pick up two antipodal 
points at $\mathrm{y}= \pm \mathrm{r}$ on such a circular orbit, (2.19) becomes,

$$
v_{1}=-v \frac{\partial \ln \rho(x, y, t)}{\partial y} .
$$

We can carry through an almost identical argument for the second component of the average momentum velocity field $v_{1} \underline{\underline{i}}-v_{2} \underline{j}$ to obtain

$$
v_{2}=-v \frac{\partial \ln \rho(x, y, t)}{\partial x} \text {. }
$$

(2.20) and (2.21) relate the probability density $\rho$ and the local average velocity field $v_{1} \underline{i}+v_{2} \underline{j}$ which holds for the quantum fluid equivalent. From (2.13) and (2.14) we can make another deduction by observing that

$$
\frac{u_{1}(x, y+r)-u_{1}(x, y-r)}{2 r}=\lambda .
$$

We can use the distribution function $f(\lambda)$ of (2.7) and $P(r)$ of (2.12) to form an average of the functional (2.22). We get

$$
\int_{0}^{\infty} \lambda f(\lambda) d \lambda=\int_{0}^{\infty} \frac{\left[u_{1}(x, y+r)-u_{1}(x, y-r)\right]}{2 r} P(r) d r
$$

or

$$
\bar{\lambda}=\int_{0}^{\infty} \frac{\left[u_{1}(x, y+r)-u_{1}(x, y-r)\right] P(r) d r}{2 r}
$$

We recall the fluid theory [9] relation between local angular velocity $\bar{\lambda}$ and the vorticity vector $\underline{\zeta}=\nabla \wedge \underline{v}=-2 \bar{\lambda} \underline{k}$ in two dimensions, for clockwise rotation,

$$
\bar{\lambda}=-\frac{1}{2}\left(\frac{\partial v_{2}}{\partial x}-\frac{\partial v_{1}}{\partial y}\right)
$$

We now impose on the momentum velocity field $\overline{\mathrm{v}}$ the two conditions,

$$
\nabla \wedge \underline{\bar{v}}=\nabla \cdot \underline{\bar{v}}=0,
$$

which together have the consequence that the two components of the mass velocity field $\underline{v}=v_{1} \underline{i}+v_{2} \underline{j}$ satisfy the Cauchy-Riemann conditions,

$$
\begin{aligned}
& \frac{\partial v_{1}}{\partial x}=\frac{\partial v_{2}}{\partial y} \\
& \frac{\partial v_{1}}{\partial y}=-\frac{\partial v_{2}}{\partial x}
\end{aligned}
$$

Thus from (2.25)

$$
\bar{r} \quad=\frac{\partial v_{1}}{\partial y}
$$


and also

$$
\bar{\lambda}=-\frac{\partial v_{2}}{\partial x}
$$

From (2.24) and (2.25), we then have,

$$
\bar{\lambda}=\frac{\partial v_{1}}{\partial y}=\int_{0}^{\infty} \frac{\left[u_{1}(x, y+r)-u_{1}(x, y-r)\right] P(r) d r}{2 r}
$$

or defining $u_{2}$ in a similar manner to $u_{1}$ as in (2.13) and (2.14) but instead of $v_{1}$ using $v_{2}$, we have

$$
\bar{\lambda}=-\frac{\partial v_{2}}{\partial x}=-\int_{0}^{\infty} \frac{\left[u_{2}(x+r, y)-u_{2}(x-r, y)\right] P(r) d r}{2 r} .
$$

However, besicies $(2.20),(2.21),(2.27)$ and $(2.28)$, all that is needed to derive Schrödinger quantum mechanics for one dimension is an equation of continuity. It i.is sufficient to take this to be an equation of the form,

$$
\frac{\partial \rho}{\partial t}=-\frac{\partial\left(\rho v_{1}\right)}{\partial x}+\Gamma
$$

$\Gamma$ in (2.33) is a function representing the transformation of fluid from the $y-$ directional flow into the $x$-directional flow or vice versa. On the $y=0$ axis, generally $\Gamma=0$ and then (2.33) reduces to the usual quantum equation of continulty for one dimension. Briefly, the Schrödinger equation can be deduced by the following steps. From $(2.20),(2.21),(2.27)$ and $(2.28)$, it follows that on the $x, y$ plane lnp satisfies Laplace's two dimensional equation,

$$
\nabla^{2} \ln \rho=0 \text {. }
$$

Consequently the real function $\rho(x, y, t)$ has the form

$$
\rho=\psi^{*}(x-i y, t) \psi(x+i y, t) \text {. }
$$

It follows from $(2.20)$ that $\left.v_{1}(x, y, t)\right|_{y}=0$ is the usual quantum current on the $y=0$ boundary. Using $\left.\rho(x, y, t) \quad\right|_{y=0}$ and $\left.v_{1}(x, y, t) \quad\right|_{y=0}$ with (2.33) for $y=0$, it is easy to confirm that $\left.\psi(x+i y, t)\right|_{y=0}=\psi(x, t)$ satisfies Schrödinger's equation on the $y=0$ boundary [2]. However, it should be observed that using (2.20) in (2.33), we obtain the equation, 


$$
\frac{\partial \rho}{\partial t}=v \frac{\partial^{2} \rho}{\partial x \partial y}
$$

for the situation or regions where $\Gamma=0 .(2.36)$ is the basic differential equation for the extended quantum density $\rho(x, y, t)$ and comparing with the equation (1.3) for Brownian motion, we can see that (2.36) is likely to have a very different physical and probabilistic interpretation from that of the Brownian motion equation.

\section{CONCLUSIONS.}

The work of the previous sections relates the Schrödinger quantum probability density to a stochastic process by a two stage procedure. The stochastic process is essentially a two dimensional process concerning states of angular velocity of fluid particles locally. The first stage lies in assuming that angular velocity states occur in angular velocity space with a probability density $f(\omega)$. We have not needed to give an explicit expression for $f(\omega)$ in order to carry through the argument. It is not clear that there is necessarily a unique $f(\omega)$ that is needed to fill this role. A decision on this last question would depend on making a more detailed study of possible stochastic structures [10] that would be consistent with the scheme we have given. However, one rather simple and interesting possibility is to use the exponential distribution,

$$
f(\omega)=\frac{e^{-\omega /|\bar{\omega}|}}{|\bar{\omega}|}
$$

where $\bar{\omega}=\frac{1}{2} \nabla \wedge \underline{\mathrm{v}}$. In this case (2.7) and (2.8) become identities and $P(r)$ can easily be found from (2.12). Also by, transforming to velocity space by (2.9) and (2.15), we find that $f(\omega)$ is transformed into the local Maxwell distribution for the velocity field $\underline{u}-\underline{v}$, relative the the mean motion $\underline{v}$. Thus the stochastic process for the relative motion has much the same character as a Maxwel1 gas. Physically we can regard the stochastic process as describing the tendency of the basic two dimensional fluid to flow into various vorticity field configurations. This can be taken to be a purely statistical matter in the absence of detailed knowledge of the underlying mechanisms of the process. The second stage is discussed briefly here because it has been given in detail elsewhere [7] but, in fact, 
relates the two dimensional flow process to the one dimensional Schrödinger equation. The most important step exhibited in the discussion here is to show that the probability density $\rho(x, y, t)$, which goes over to the quantum $\rho(x, t)$ when $y \rightarrow 0$, apart from dimensioned numerical multipliers, once more relates probability to time in orbit as is the case the probability density in some classical contexts. It also seems from this work and earlier work [1] that ordered rotatory motion has a strange capacity to simulate the random heat-like forms of kinematic activity. ACKNOWLEDGEMENT. I am grateful to Professor N.G. van Kampen for a number of useful criticisms that have led to clarifications and an improvement in the presentation of this work.

\section{REFERENCES}

1. BARTLETT, M.S. An Introduction to Stochastic Processes, Cambridge Univ. Press., (1967).

2. GILson, J.G. On Stochastic Theories of Quantum Mechanics, Proc. Camb. Phil. Soc., 64 (1968), 1061-1070.

3. DE LA PENA-AUERBACH, L. and CETTO, A.M. Quantum Mechanics derived from Stochastic Electrodynamics, Found. Phys. 으, Nos. 3,4, April (1978) 191-210.

4. NELSON, E. Derivation of the Schrödinger Equation from Newtonian Mechanics, Phys. Rev. 150, No. 4, (1966) 1079-1085.

5. ROY, S. Relativistic Brownian Motion and the Foundations of Quantum Mechanics, Nuovo Cim. 51 B, N, 1, 11 Maggio (1979) 29-44.

6. HAKIM, R. Relativistic Stochastic Processes, J. Math. Phys. 9, No. 11, Nov . (1969) 1805-1818.

7. GILSON, J.G. The Fluid Process Underlying Quantum Mechanics, Acta Phys. Hung. Tomus 44, No. 4, (1978) 333-352.

8. GILSON, J.G. Subquantum Thermodynamics, Acta Phys. Polonica A52, (1977).

9. MILNE-THOMSON, L.M. Theoretical Hydrodynamics, Macmillan London, (1949) 315.

10. VAN KAMPEN, N.G. Stochastic Differential Equations, Physics Reports (Section C of Physics Letters) 24, No. 3, (1976) 172-?28.

11. GILSON, J.G. Statistical Relativistic Particle, J. App1. Prob. 4, (1967) 389-396. 


\section{Mathematical Problems in Engineering}

\section{Special Issue on}

\section{Time-Dependent Billiards}

\section{Call for Papers}

This subject has been extensively studied in the past years for one-, two-, and three-dimensional space. Additionally, such dynamical systems can exhibit a very important and still unexplained phenomenon, called as the Fermi acceleration phenomenon. Basically, the phenomenon of Fermi acceleration (FA) is a process in which a classical particle can acquire unbounded energy from collisions with a heavy moving wall. This phenomenon was originally proposed by Enrico Fermi in 1949 as a possible explanation of the origin of the large energies of the cosmic particles. His original model was then modified and considered under different approaches and using many versions. Moreover, applications of FA have been of a large broad interest in many different fields of science including plasma physics, astrophysics, atomic physics, optics, and time-dependent billiard problems and they are useful for controlling chaos in Engineering and dynamical systems exhibiting chaos (both conservative and dissipative chaos).

We intend to publish in this special issue papers reporting research on time-dependent billiards. The topic includes both conservative and dissipative dynamics. Papers discussing dynamical properties, statistical and mathematical results, stability investigation of the phase space structure, the phenomenon of Fermi acceleration, conditions for having suppression of Fermi acceleration, and computational and numerical methods for exploring these structures and applications are welcome.

To be acceptable for publication in the special issue of Mathematical Problems in Engineering, papers must make significant, original, and correct contributions to one or more of the topics above mentioned. Mathematical papers regarding the topics above are also welcome.

Authors should follow the Mathematical Problems in Engineering manuscript format described at http://www .hindawi.com/journals/mpe/. Prospective authors should submit an electronic copy of their complete manuscript through the journal Manuscript Tracking System at http:// mts.hindawi.com/ according to the following timetable:

\begin{tabular}{|l|l|}
\hline Manuscript Due & March 1, 2009 \\
\hline First Round of Reviews & June 1, 2009 \\
\hline Publication Date & September 1,2009 \\
\hline
\end{tabular}

\section{Guest Editors}

Edson Denis Leonel, Department of Statistics, Applied Mathematics and Computing, Institute of Geosciences and Exact Sciences, State University of São Paulo at Rio Claro, Avenida 24A, 1515 Bela Vista, 13506-700 Rio Claro, SP, Brazil; edleonel@rc.unesp.br

Alexander Loskutov, Physics Faculty, Moscow State University, Vorob'evy Gory, Moscow 119992, Russia; loskutov@chaos.phys.msu.ru 